\title{
Therapies targeting FLS and TNF can have synergistic effects
}

The combination of DMARDs and drugs that target fibroblast-like synoviocytes (FLS) has been proposed as an approach to improve therapeutic response in rheumatoid arthritis (RA), but agents that selectively target FLS are currently lacking. New esearch suggests that receptor-type tyrosine-protein phosphatase $S$ (PTPRS), which is highly expressed on FLS, could be leveraged to develop an FLS-targeted agent for use in combination therapy for RA.

The researchers had previously shown that targeting PTPRS on FLS using the decoy protein immunoglobulin-like domains 1 and 2 (Ig1\&2), which activates the phosphatase, impaired FLS invasiveness into cartilage and reduced the development of FLS-mediated inflammatory arthritis in mice. The current work explored whether the mechanism of action of Ig $1 \& 2$ in arthritis is mediated through suppression of immune cell function and also evaluated the effects of using Ig1\&2 in the context of TNF inhibition.

"We discovered that PTPRS is enriched in synovial lining layer RA FLS, a population identified as critical for RA pannus invasiveness," reports Mattias Svensson, first author of the study published in Science Advances. Notably, they showed that Ig1\&2 inhibited the motility of RA FLS but not osteoarthritis FLS.

The researchers next showed that the anti-arthritic effect of Ig1\&2 in mice with $\mathrm{K} / \mathrm{B} \times \mathrm{N}$ serum transferinduced arthritis or collagen-induced arthritis (CIA) is not mediated through inhibition of adaptive or innate immune cells.

"We unexpectedly found that TNF reduces the expression of PTPRS in RA FLS," says corresponding author Nunzio Bottini. "This discovery led us to a finding that in our opinion is very exciting, which is a therapeutic synergy between suboptimal doses of $\operatorname{Ig} 1 \& 2$ and of anti-TNF therapy

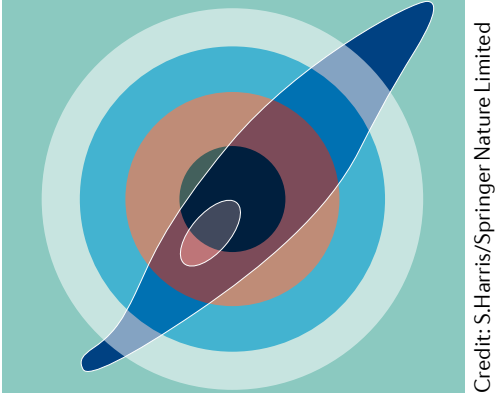

in reversing arthritis in the CIA model." As well as reversing clinical disease, the combination therapy also protected against bone erosion and cartilage loss, whereas these effects were not seen with either agent administered as monotherapy at suboptimal doses.

The researchers are planning further studies to better understand how activation of PTPRS on FLS leads to substantial anti-inflammatory effects in arthritic joints and to further establish the in vivo safety of Ig $1 \& 2$ and potentially of other approaches to activate PTPRS.

Sarah Onuora

ORIGINAL ARTICLE Svensson, M. N. D. et at. Synoviocyte-targeted therapy synergizes with TNF inhibition in arthritis reversal. Sci. Adv. 6, eaba4353 (2020)

\section{Autoantibodies disrupt muscle repair and promote IIM progression}

New research suggests that tripartite motif (TRIM) family proteins act as autoantigens in idiopathic inflammatory myopathy (IIM) and contribute to disease progression via a positive feedback loop. The findings of the study suggest that antibodies against these proteins compromise muscle membrane repair, and decreased sarcolemmal integrity leads to aberrant autoantigen presentation that promotes autoimmunity.

The research focused on the protein TRIM72 (also known as MG53), which had been previously identified

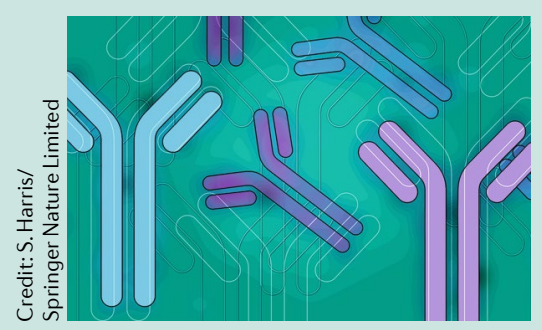

as an important component of the sarcolemmal repair process in striated muscle. "We identified autoantibodies targeting TRIM72 [...] in a subset of myositis patient sera and established a direct causal effect of these antibodies on defective membrane repair," says corresponding author Noah Weisleder. "To our knowledge, we are the first to identify this autoantibody and directly demonstrate an effect on myositis disease progression."

The researchers demonstrated that adoptive transfer of lymph node cells from Foxp $3^{-/ r}$ Syt $7^{-1-}$ mice into recipient $\mathrm{Rag}^{-1-}$ mice resulted in severe inflammation in proximal muscle whereas distal muscle was spared, recapitulating the pattern seen in patients with IIM. Notably, distal skeletal muscle had impaired membrane integrity and reduced capacity for membrane repair following injury in the absence of inflammation.
Autoantibodies against TRIM72 were detected in sera from Foxp $3^{-/ Y}$ Syt7 $7^{-1-}$ mice and in adoptive transfer recipient mice, as well as in sera from patients with dermatomyositis or polymyositis. In healthy skeletal muscle subjected to laser injury, the presence of patient sera with high levels of anti-TRIM72 autoantibodies suppressed sarcolemmal resealing, whereas this repair capacity was rescued in the presence of these same serum samples depleted of anti-TRIM72 antibodies.

"Future studies will determine if other proteins involved in the cell membrane repair response are also autoantigen[s] and to what extent these autoantigens [contribute to] disease progression," says Weisleder. "If cell membrane integrity can be increased therapeutically," he adds, "it may be possible to limit the extent of muscle injury and wasting caused by autoimmune diseases targeting skeletal muscle."

Sarah Onuora

ORIGINAL ARTICLE McElhanon, K. E. et al. Autoantibodies targeting TRIM72 compromise membrane repair and contribute to inflammatory myopathy.J. Clin. Invest. 6, eaba4353 (2020) 